\title{
EL FÚTBOL EN TRES TIEMPOS SOCIALES: OBSERVACIONES DE UN FESTIVAL ASOCIATIVO EN CAJU, RÍO DE JANEIRO, BRASIL 2014
}

\author{
${ }^{1}$ Fernando Segura M. Trejo \\ ${ }^{2}$ Adriana Islas Govea
}

\begin{abstract}
RESUMEN
El objetivo del artículo radica en exponer avances de investigación sobre el deporte, el fútbol en particular, en tanto herramienta de trabajo social. Con base en la observación del festival Football for Hope Caju 2014 desarrollado durante el Mundial de Brasil en la zona portuaria de Rio de Janeiro en julio de 2014, se busca aquí reflexionar sobre el uso del deporte para fines sociales. A partir de un marco de referencia general, emanado de investigaciones internacionales ya realizadas, el foco de atención se extiende desde los relatos de los participantes entrevistados de la delegación de Ecuador, la observación etnográfica del festival y su metodología conocida como Fútbol 3 Tiempos. Por este medio, el artículo apunta a un análisis de las percepciones del pasado, el momento del festival y las expectativas de los participantes en tanto destinatarios principales del encuentro.
\end{abstract}

Palabras claves: Fútbol - festival asociativo- Ecuador- Tres Tiempos - participación - género

\section{SOCIAL SOCCER IN THREE TIMES: OBSERVATIONS OF A FESTIVAL ASSOCIATION IN CAJU, RIO DE JANEIRO, BRAZIL 2014}

\begin{abstract}
The aim of this article lies in exposing research findings about the use of sport, of football in particular, as a tool for social work. Based on the observation of the Football for Hope's festival carried out in Caju, the harbor area of Rio de Janeiro, during the World Cup of Brazil 2014, social accompaniment through football is discussed. Taking into consideration other international researches already conducted, the data here presented emanates from the narratives of the participants interviewed and ethnographic observation of this festival that is characterized by the use of a kind of football with three halves. The article reviews the participants' perceptions about their past, the experience of the festival and their expectations as the ultimate recipients.
\end{abstract}

Keywords: Football - associative festival - Ecuador - three halves - participation - gender

\footnotetext{
${ }^{1}$ Doutor em Sociología pela Escola de Altos Estudos em Ciências Sociais de Paris, França. Professor Visitante na PósGraduação de Sociologia da Universidade Federal de Goiás - UFG, (Brasil). Pesquisador filiado ao Centro de Investigación y Docencias Económicas (CIDE) no México. Pesquisador filiado ao laboratório Violence, Identitités Politiques et Sport, Université de Rennes II na França. E-mail: fernando.segura@ cide.edu ${ }^{2}$ Mestranda em História Internacional pelo Centro de Investigación y Docencias Económicas (CIDE). Socióloga pela Universidade Nacional Autónoma - UNAM, (México). E-mail: aeigovea@gmail.com
} 


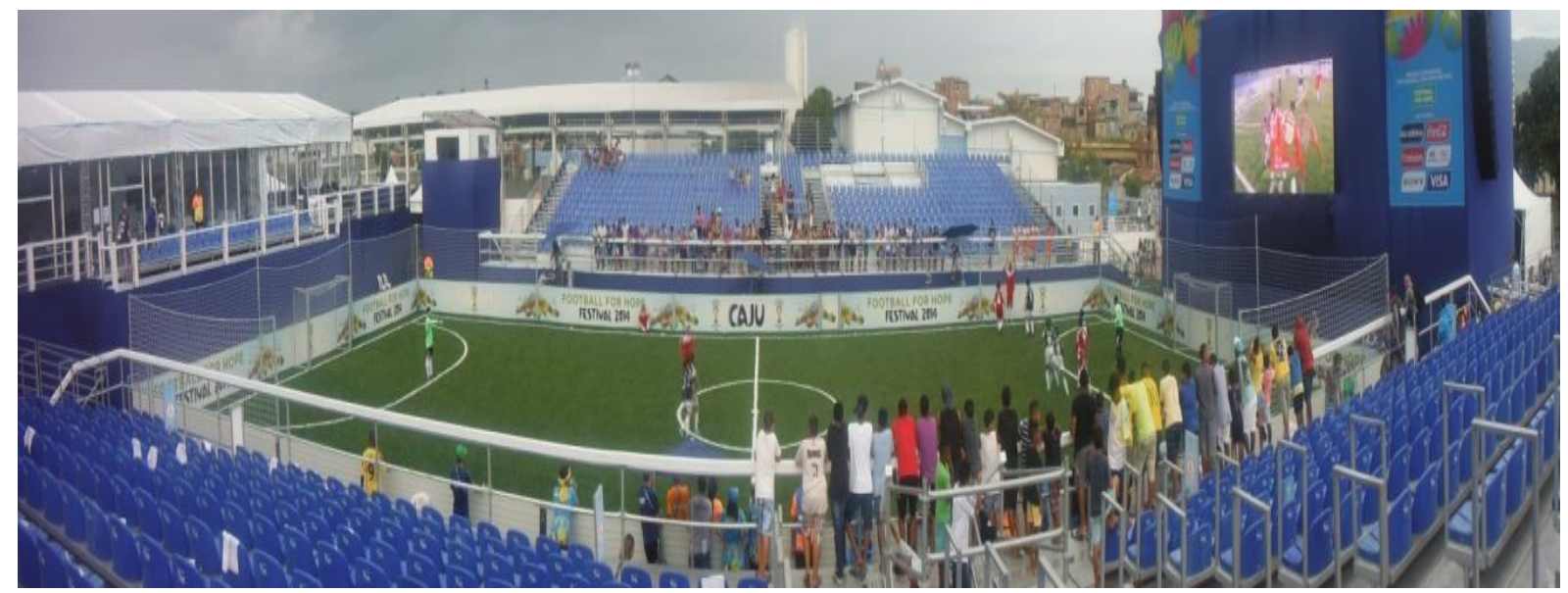

Foto: Autores

\section{INTRODUCCIÓN}

El fútbol al que en este artículo se hace referencia tiene que ver con una forma alejada de su versión profesional. Pero a su vez, tampoco se remite a una práctica amateur en su esquema habitual, donde normalmente un equipo gana en la cancha por los goles convertidos. Esto sería normal en todo encuentro futbolístico, en cualquier liga profesional, semiprofesional, en espacios amateurs, torneos, ligas barriales, inclusive en partidos de potrero (Archetti, 2003), o bien, en un simple encuentro entre amigos. Nos ocuparemos, aquí, de una modalidad en donde los puntos vienen dados por el comportamiento y el respeto a las reglas definidas por los propios participantes, en una suerte de evaluación y negociación posterior a la interacción en el campo de juego. Esta metodología, conocida como Fútbol Tres Tiempos, procura desarrollar en los jóvenes practicantes el hábito del análisis de situaciones inmediatas y el accionar, en el día a día, para favorecer la resolución de problemas, la toma de decisiones concertada así como la elaboración de proyectos de vida.

El objetivo de este artículo apunta, así, hacia una contribución empírica producto de la observación de campo y una serie entrevistas con protagonistas de un festival internacional articulado en la metodología del Fútbol 3 Tiempos. Para este cometido de investigación cualitativa, los autores se apoyan en dos nociones emprestadas a Erving Goffman: la manera en la cual los entrevistados se presentan a sí mismos (Goffman, 1959) y la forma en la cual perciben, analizan y comentan el marco de referencia (Goffman, 1974) en el que están actuando (Goffman, 1959).
Así, en concordancia con la lógica del festival observado en la zona de Caju, Río de Janeiro, en julio 2014, se propone dividir el horizonte de análisis en tres tiempos a partir de los relatos recabados. Una revisión del recorrido de los participantes, cuyo resultado se expresa en este encuentro internacional como segundo momento importante, y finalmente, un tercer horizonte que se refiere tanto a su futuro, plasmado en sus expectativas y proyecciones. El artículo propone, también, una revisión de su situación a más de un año del acontecimiento.

El marco en el que este artículo se inserta, por lo tanto, abarca un debate más amplio sobre el uso del deporte como elemento de desarrollo social. En ese sentido, el objetivo paralelo (Becker, 1960) es proporcionar evidencia de investigación, en español, acerca del estudio del deporte, y el fútbol en específico, en tanto herramienta diseñada para el trabajo social. Más específicamente, los autores desean responder a las siguientes preguntas: ¿qué sucede con los participantes consultados en este proceso que lleva al festival? ¿Qué ocurre durante el evento? ¿Qué pasa después del acontecimiento con el transcurrir de los meses? Como lo enfatiza Giulianotti $(2005 ; 2011)$ el principal indicador de impacto de este tipo de programas necesita remitirse a las percepciones de los propios destinatarios.

\section{El Marco del Deporte como Objeto de Desarrollo} Social

Un importante número de actores internacionales y locales, entre los cuales las organizaciones no gubernamentales (ONGs), con apoyos diversos de instituciones universitarias, 


\section{El Fútbol en Tres Tiempos Sociales: Observaciones de Un Festival Asociativo en Caju, Río de}

Janeiro, Brasil 2014

gobiernos e iniciativas privadas ha llegado a constituir una tendencia denominada Sport for Development and Peace (SDP) (Kidd, 2008) desde la década de 1990 y en particular el inicio del nuevo milenio. El deporte para el desarrollo y la paz, en el cual el fútbol es una elección recurrente, no reviste una faceta uniforme, ya que en él ingresan intereses dispares, subsidios de federaciones deportivas, organizaciones en pos del desarrollo humano, ONGs-militantes críticas del poder establecido, hasta los más diversos intereses corporativos (Giulianotti, 2011).

Los programas se han multiplicado en los años recientes y sus participantes han crecido en un número considerable. Los proyectos son destinados a grupos en situación de riesgo y diferentes formas de exclusión social con el objetivo de ofrecer espacios y herramientas para la inclusión y el desarrollo de capacidades. Entre los efectos sociológicos que han sido atribuidos a diferentes programas, se ha podido identificar aumentos del capital social, es decir, de lazos sociales positivos para los participantes. En iniciativas de clubes sociales y deportivos en Sudáfrica (Burnett, 2006) la frecuencia de asistencia a espacios que fomentan diálogo y participación, o la extensión de acceso a informaciones $y$ servicios sociales para jóvenes confrontados a precariedad urbana en Rotterdam, Holanda (Spaaij, 2009) conforman parte de la evidencia académica recabada.

Otras investigaciones, como la del proyecto $\mathrm{STAR}^{3}$, creado para la reconciliación posterior a la guerra civil en Liberia han mostrado fuertes contradicciones entre los objetivos de paz y la dimensión competitiva del fútbol, la cual, en lugar de propiciar una interacción distendida, demostró generar tensiones entre los grupos de participantes (Roowdook \& Palmer, 2011). Estudios sobre un programa para la paz entre jóvenes israelitas y palestinos ha dejado entrever las dificultades de integrar mujeres, así como el desafío de la continuidad frente a escenarios de conflicto en la región (Sudgen, 2008).

Sobre el papel de proyectos exclusivos para mejorar el auto-estima de mujeres en contextos de vulnerabilidad, Jeanes (2013) ha discutido el empoderamiento de mujeres en un programa de Zambia y la posibilidad de incluir objetivos de salud pública en paralelo a programas de actividad física.

El panorama, el repertorio de programas y enfoques existentes son en efecto muy amplios dado que varios festivales y torneos en el mundo celebran el uso del deporte, y del fútbol en particular, como una herramienta de cambio social. Ese es el caso, por ejemplo, del mundial Homeless World Cup, creado en 2003 y desarrollado todos los años en una ciudad

\footnotetext{
${ }^{3}$ Por sus siglas en inglés, Self-discipline, Truthfulness, Appreciation and Respect (STAR) fue lanzado en el año 2006 por la ONG Samaritan's Purse (Rookwood and Palmer, 2011: 196).
}

diferente. Algunas investigaciones han destacado, precisamente, la creación de capital social para jugadores de este torneo (Sherry, 2010), así como la constitución de objetivos en los jugadores (Sherry et al, 2013).

Ahora bien, cabe preguntarse sobre las modalidades y las formas que el deporte asume en estos programas y eventos. ¿Qué tipos de modalidades deportivas son introducidas y cuáles son sus objetivos? ¿Hasta qué punto y de qué manera el deporte, y el fútbol en particular, estimula el desarrollo social? Como entender el desarrollo social, depende del contexto donde se introducen programas (Burnett, 2009). Además, Coalter (2010) subraya el hecho que las investigaciones sobre este recurso del deporte deben enfocarse en las situaciones de los participantes, independientemente de los discursos de institucionales, e inclusive, de las exigencias de los patrocinadores. Trabajos de acompañamiento han encontrado que, a pesar de convertirse en una experiencia estimulante, la naturaleza competitiva de un evento puede volverse tensa para aquellos que sufren derrotas abultadas en torneos donde existen brechas considerables en los niveles de los equipos (Magee, 2011). Esto demuestra que la observación de un mundial como el Homeless abre interrogantes acerca de las experiencias vividas de acuerdo con la victoria o la derrota en los campos de juego (Magee \& Jeanes, 2013; Segura M. Trejo, 2011; 2013a; 2013b, Segura et al, 2015).

Cuando se estiran los criterios basados en el mérito deportivo en programas sociales, la integración de poblaciones fragmentadas o grupos socialmente vulnerables se ve limitada (Attali, 2004). Así, esto puede conllevar hacia la potencial exclusión de destinatarios, en particular aquellos participantes menos dotados de habilidades deportivas y físicas o inclusive exclusiones de género (Gasparini \& Marchiset, 2008).

En este sentido, dos grandes modalidades ordenan el uso del fútbol como herramienta social. Una primera que puede considerarse como competitivamotivacional, y en donde a pesar de circunscribirse a un uso social, la dinámica clasifica a los mejores equipos a partir del rendimiento deportivo. Los partidos los gana aquella escuadra que marca más goles (Segura M. Trejo, 2014b; Segura et al, 2015). La otra modalidad se puede denominar socio-educativa y sus características se distinguen por hacer de los objetivos educativos una dimensión que supere a la utilización del fútbol, donde los festivales son solamente un instante y no un fin en un proceso de reclutamiento.

En este escenario internacional, los autores entraron en contacto con la red de Streetfootballworld, fundada en 2002, articuladora de varias ONGs pioneras en este movimiento, en una suerte de continuación del proyecto Fútbol por la Paz surgido en Medellín, Colombia; de Defensores del Chacho en Argentina, y The Matare Youth Sport Association, en Nairobi, 
Kenia, entre otras. Esta red suscribió una alianza desde el año 2006 con el programa de responsabilidad social de FIFA, Football for Hope. Durante el mundial de Alemania 2006 se realizó en Berlín un festival internacional, replicado en Sudáfrica 2010 en Johannesburgo. La siguiente cita fue fijada para el Mundial de Brasil 2014 y Río de Janeiro fue designado como escenario para el festival Football for Hope 2014.

Con el objetivo de poder hacer una observación de campo en una cita con delegaciones de todos los continentes, los autores solicitaron desde el año 2012, la posibilidad de asistir y observar, para propósitos de investigación, el festival que se realizaría en 2014 en Rio de Janeiro. Uno de los autores se encontraba ya en Brasil con apoyo del Consejo Nacional de Ciencia y Tecnología (CONACYT) de México en estudio de campo con una serie de organizaciones locales (Segura M. Trejo, 2014a; Segura M. Trejo, 2014c). Concedido el acceso para Football for Hope 2014, el desafío pasaba por definir qué aspectos observar en un microcosmos de 32 delegaciones entre el 3 y el 10 de julio de 2014.

\section{METODOLOGÍA DE OBSERVACIÓN Y ANÁLISIS}

Las dos principales herramientas de recolección de datos para esta investigación fueron la observación del festival, desde su inicio hasta su final, así como la realización de entrevistas con participantes. De la misma forma que otros trabajos han recurrido a esta estrategia de investigación para festivales asociativos de fútbol para fines sociales (Sherry, 2010; Sherry et al, 2011; Magee, 2011; Gannet et al, 2014) se eligió, así, seguir el festival a través una delegación en particular, en este caso aquella proveniente de Ecuador, debido a la apertura con sus responsables, en función de un previo encuentro en un festival latinoamericano desarrollado en Salvador de Bahía en diciembre 2013 en el que se había podido observar la dinámica de este fútbol en tres tiempos (Segura M. Trejo, 2014b) y conocer, así, a uno de los responsables ecuatorianos.

El foco para la observación de Football for Hope 2014 en Caju, consistió en acompañar la experiencia, los momentos particulares y las situaciones de interacción de los jóvenes participantes en su propio marco de referencia (Goffman, 1974). Las entrevistas con los seis participantes, tres varones y tres chicas de entre 15 y 18 años, fueron convenidas con los responsables y cada participante fue consultado para acceder a las charlas personales. Los participantes serán referidos en adelante como Participante 1- 6 . Asimismo, dos jóvenes líderes que actuaron como mediadores fueron también entrevistados acerca de su experiencia en el festival.
Para acceder a las entrevistas, se pidió la autorización de los dos responsables de la delegación en el primer día del festival. Una vez avalado el pedido, los dos autores fueron presentados con los participantes, como investigadores interesados en conocer la experiencia del festival. De ahí, se procedió a un acercamiento en los primeros momentos de aclimatación, es decir, actividades lúdicas con los mediadores, como rondas de expresión corporal y canciones. Sin participar de las mismas, los autores pudieron conseguir un diálogo natural con los jóvenes. En un segundo momento, a partir del tercer día, se solicitó a los propios participantes un momento durante el festival para realizar una entrevista con cada uno.

Para este cometido, se decidió entre los autores, que quien realizaría las entrevistas sería la coautora, por una cuestión de mayor proximidad, con el fin de estimular la empatía con los jóvenes. Se pensó así, porque el autor podría ser percibido como un investigador, de una diferencia de edad mayor y podrían asociar la entrevista a una evaluación, o comportarse con menos naturalidad. Las entrevistas se efectuaron en diferentes momentos, en promedio una o dos por día, generalmente después de los almuerzos o descansos por la tarde y fueron grabadas en un celular con el consentimiento de los entrevistados. Las mismas apuntaron a conocer el recorrido de los jóvenes, sus vidas y su ingreso al proyecto local en Ecuador, con énfasis en el proceso que los llevó a convertirse en participantes de la delegación que viajaría a Río de Janeiro en julio 2014. En esos recuentos se procuró dialogar sobre los significados otorgados a la experiencia.

Las entrevistas fueron complementadas por la observación de los instantes de definición de reglas previas a los partidos de fútbol con las delegaciones que fueron apareciendo en el recorrido de los jóvenes de Ecuador. Durante los encuentros deportivos los autores se posicionaban en las gradas o detrás de uno de los arcos para acompañar las acciones en el campo de juego y poder conversar acerca de ellas, posteriormente, con los protagonistas y responsables de la delegación. Muchas veces se les consultaba, $a$ posteriori, sobre la aplicación o la interpretación de situaciones específicas.

Estas conversaciones complementaron las entrevistas en lo que a la recolección de datos se refiere, ya que constituían percepciones espontáneas de los participantes sobre la experiencia del festival, es decir el marco de referencia (Goffman, 1974) y sus diferentes momentos, los primeros tiempos, los encuentros deportivos y los espacios de discusión. Así, la grilla de observación prestó atención a los denominado terceros tiempos, es decir, los espacios reservados para asignar los puntajes a los partidos desarrollados, en donde las delegaciones debían evaluar el comportamiento de ambas partes, su propia actuación y la del equipo contrario. Fue clave, por lo 
tanto, prestar atención a las situaciones de argumentación, construcción de consensos y pedido de explicaciones en algunos casos.

El seguimiento de la delegación reservó tiempo para compartir los almuerzos. Para esto, se tuvo que pedir autorización y acreditación a los organizadores del festival Football for Hope. Durante esos instantes, las opiniones y las conversaciones, en un tono de distancia y distensión respecto a los momentos de mayor atención a las normas de los encuentros deportivos, permitió una convivencia y un acercamiento más íntimo con el grupo. La toma de notas y apuntes resulta siempre fundamental en este proceso de observación (Lofland \& Lofland, 1995), pues además de ofrecer una guía, permitía registrar a los dos autores, en cuadernos diferentes, los eventos y las expresiones considerados como significativos. Tales apuntes daban la posibilidad de comentar las observaciones al final de la jornada. Asimismo, este material sirvió para el análisis en retrospectiva, pasados los meses, para escribir el artículo.

Posteriormente, ya en una segunda etapa de procesamiento de los testimonios obtenidos, se procedió a la desgravación de las entrevistas para su análisis y la consulta con los responsables de la delegación acerca de la situación de los participantes más de un año del acontecimiento. El contacto con la asociación de Ecuador permitió varias verificaciones de informaciones y balances de los participantes a uno y dos años del acontecimiento. Sin estas consultas con la fundación Fudela, no hubiera sido posible estimar el devenir y el impacto en los participantes.

Exposición de la Investigación realizada en el Festival de Caju 2014
El primer elemento conceptual, como referencia organizadora de la experiencia de los participantes (Goffman, 1974) en este festival, se trata de la modalidad específica de fútbol en tres tiempos. Bajo este esquema, dentro de la categoría que denominamos como uso socio-educativo del fútbol, son los propios participantes quienes definen las reglas con las cuales jugarán sus partidos.

Los equipos se ponen de acuerdo sobre los saques de banda, los tiros de esquina, o en otro nivel de profundidad, que el gol debe ser válido solamente si una de las participantes participa en la jugada. En el mismo sentido, puede acordarse que los dos equipos tienen que festejar el gol del contrario o que el gol de una mujer vale doble. Las posibilidades son múltiples y quedan abiertas a la creatividad y a las estrategias, también. Posteriormente se realizan los partidos sin árbitros y los participantes tienen que adecuarse a lo convenido en el segundo tiempo de juego, es decir, los partidos.

En el tercero, los participantes negocian la atribución de puntajes, para los cuales consideran no solamente los goles sino los comportamientos, puestos en debate, defendidos o cuestionados con posturas y argumentos. Pueden darse situaciones en las cuales aquel equipo que marcó más goles no termine ganando el encuentro. Fue precisamente el acceso a la delegación lo que nos permitirá reflexionar sobre las trayectorias de los jugadores consultados y el sentido otorgado a esta modalidad. El siguiente cuadro muestra un mapa de las principales variables que ordenaron las entrevistas y aparecieron como relevantes en las conversaciones.

\section{Cuadro I. Percepciones del impacto en los participantes}

\begin{tabular}{|c|c|c|c|c|c|}
\hline & $\begin{array}{l}\text { Impacto } \\
\text { reconocido }\end{array}$ & $\begin{array}{l}\text { Perspectivas de } \\
\text { estudios }\end{array}$ & $\begin{array}{l}\text { Impresiones del } \\
\text { Festival } 2014\end{array}$ & $\begin{array}{l}\text { Metodología } \\
3 \text { Tiempos }\end{array}$ & $\begin{array}{c}\text { Otros } \\
\text { proyectos a } \\
\text { futuro } \\
\end{array}$ \\
\hline Participante 1 & $\begin{array}{l}\text { Mejora en la auto- } \\
\text { estima y confianza en } \\
\text { sí Aceptar recibir } \\
\text { indicaciones }\end{array}$ & $\begin{array}{l}\text { Terminar estudios } \\
\text { secundarios }\end{array}$ & $\begin{array}{l}\text { Visto como un } \\
\text { premio al esfuerzo }\end{array}$ & $\begin{array}{l}\text { Seguir } \\
\text { participando y } \\
\text { organizar } \\
\text { torneos } \\
\end{array}$ & $\begin{array}{l}\text { Talleres de } \\
\text { Liderazgo como } \\
\text { ponente }\end{array}$ \\
\hline Participante 2 & $\begin{array}{l}\text { Aprendizaje de } \\
\text { perseverancia en metas }\end{array}$ & $\begin{array}{l}\text { Realizar estudios } \\
\text { universitarios de } \\
\text { administración }\end{array}$ & $\begin{array}{l}\text { Momento de poner } \\
\text { en práctica la } \\
\text { preparación }\end{array}$ & $\begin{array}{l}\text { Seguir con la } \\
\text { fundación y el } \\
\text { voluntariado }\end{array}$ & $\begin{array}{l}\text { Realizar talleres } \\
\text { de danza y } \\
\text { música }\end{array}$ \\
\hline Participante 3 & $\begin{array}{l}\text { Conocer nuevas } \\
\text { personas }\end{array}$ & $\begin{array}{l}\text { Deseos de estudios } \\
\text { universitarios pero } \\
\text { no sabe en cuál área }\end{array}$ & $\begin{array}{l}\text { Positivo pero evoca } \\
\text { fatiga }\end{array}$ & $\begin{array}{l}\text { Continuar } \\
\text { practicando la } \\
\text { metodología } \\
\end{array}$ & $\begin{array}{l}\text { Dice no tener } \\
\text { otros }\end{array}$ \\
\hline Participante 4 & $\begin{array}{l}\text { Aceptación del otro } \\
\text { género en el plano } \\
\text { deportivo }\end{array}$ & $\begin{array}{l}\text { Terminar estudios } \\
\text { secundarios y } \\
\text { realizar ingeniería }\end{array}$ & $\begin{array}{l}\text { Positivo y deseos de } \\
\text { participar en otros } \\
\text { festivales }\end{array}$ & $\begin{array}{l}\text { Seguir } \\
\text { practicando y } \\
\text { organizar un } \\
\text { festival barrial }\end{array}$ & $\begin{array}{l}\text { Probar suerte en } \\
\text { el fútbol } \\
\text { profesional }\end{array}$ \\
\hline & & & Positivo Pero & & \\
\hline
\end{tabular}




\begin{tabular}{|c|l|l|l|l|l|}
\hline Participante 5 & $\begin{array}{l}\text { Incorporación de la } \\
\text { metodología como una } \\
\text { formación personal }\end{array}$ & $\begin{array}{l}\text { Terminar estudios } \\
\text { secundarios }\end{array}$ & $\begin{array}{l}\text { lamenta la } \\
\text { inclinación de } \\
\text { equipos con } \\
\text { objetivos demasiado } \\
\text { competitivos }\end{array}$ & $\begin{array}{l}\text { Seguir } \\
\text { participando y } \\
\text { organizar un } \\
\text { festival }\end{array}$ & $\begin{array}{l}\text { Voluntario con la } \\
\text { Fundación }\end{array}$ \\
\hline Participante 6 & $\begin{array}{l}\text { Incorporación de } \\
\text { metas personales }\end{array}$ & $\begin{array}{l}\text { Seguir estudios de } \\
\text { medicina }\end{array}$ & $\begin{array}{l}\text { Positivo } \\
\text { Posibilidad de } \\
\text { conocer proyectos } \\
\text { internacionales }\end{array}$ & $\begin{array}{l}\text { Seguir } \\
\text { participando y } \\
\text { organizar } \\
\text { festivales } \\
\text { barriales }\end{array}$ & $\begin{array}{l}\text { Participar en } \\
\text { talleres contra } \\
\text { trata de personas }\end{array}$ \\
\hline
\end{tabular}

Construcción: Autores

Así, con este ordenamiento inicial propuesto al lector, las siguientes secciones expondrán el análisis cualitativo de las percepciones de los participantes respecto al proceso de los llevó al festival y al sentido otorgado en el momento de vivirlo. En armonía con la metodología, pero trasladada a una secuencia de hechos que conforman una trayectoria (Becker, 1963), el pasado aparece, así, como un tiempo que ha sido modificado en relación con este tipo de fútbol, basado en la construcción consensual, la práctica mixta entre géneros y el diálogo. En el análisis aquí propuesto, el segundo tiempo social se considera a la experiencia misma del festival en cuestión. Y el tercero, el horizonte de proyecciones que los participantes indicaron en sus entrevistas, en referencia a las expectativas de futuro derivadas de este viaje.

\section{PRIMER TIEMPO: PERCEPCIONES Y RELATOS DE UN INICIO}

Tal como lo expresa Goffman, las personas tienden a reconocer eventos particulares a partir de marcos de percepciones o esquemas de interpretación que les son familiares (Goffman, 1974: 21). Un marco extraordinario, como lo es un festival internacional, producto de una preparación intensa y de un viaje a Brasil implica todo un proceso de construcción y otorgamiento de sentidos. Las expresiones de los seis participantes destacan el inicio de la metodología tres tiempos desarrollada por la ONG, Fudela, como el ingreso hacia un aprendizaje sobre diferencias y una adaptación a un tipo de fútbol mixto.

Una de las jugadoras explicó que al entrar al programa debió aprender a aceptar indicaciones. La llegada al proyecto se relata, por cada uno de los entrevistados, como el comienzo de un proceso de cambio. Tal como se expuso en el cuadro I anterior, la cuestión de género surge como una de las principales variables evocadas en este recorrido. En este sentido, si bien algunas de las jugadoras y los jugadores ya habían participado de encuentros mixtos, esto no era un hábito, especialmente para los jóvenes. La Participante 1 relató haberse: "acercado al fútbol a través de mis hermanos, con quienes empecé a jugar". Sin embargo, su relato da cuenta que: "Muchas veces me decían que como mujer no podía jugar al fútbol en los partidos barriales. Me decían que el fútbol no era para niñas. Pero luego, a la hora de jugar, algunos lo hacían bruscamente para amedrentarme y mostrarme que era cosa de hombres. Pero nunca desistí". La
Participante 2 rememoró: "Como chica me tuve que acostumbrar a los pelotazos que recibía de los varones".

Para los jóvenes de la delegación, el habituarse a jugar con jugadoras en forma recurrente formó parte de un proceso de cambio de percepciones. Participante 4: "Al principio, no me resultaba cómodo jugar con chicas, consideraba que ellas no estaban al mismo nivel. Es cierto que existe una diferencia física que no puede ser ignorada. Pero la metodología 3 Tiempos permite ver las cosas de otra manera. No estás ahí para competir o para exigirle a tu compañero o compañera que sea el mejor con el balón, sino más bien para alentarlo y para adaptarte, tú, a los niveles de los demás".

La interacción con el otro género y la modificación de actitudes se hace palpable en la descripción. El Participante 5: "Realmente necesité tiempo para asimilar esta metodología. Confieso que en un inicio no la entendía. No sabía para qué me podía servir. Yo solamente quería jugar al fútbol. Después fui entendiendo que me servía para mi formación personal". Algunos de los participantes indicaron que varios jóvenes conocidos desistían de permanecer en Fudela, debido a que buscaban un fútbol más parecido a sus aspiraciones de competencia y esquemas uniformes en cuanto a género: fútbol masculino por un lado y femenino, por otro. En ese sentido, la Participante 3 agrega: "Había una época en la cual me aburría, eran demasiadas charlas que recibíamos. Pero poco a poco me fui dando cuenta que el tratamiento que nos daban a las chicas era menos agresivo en el cancha. Ahí me di cuenta que las charlas estaban cambiando la dinámica y las reglas ayudan a integrar a las chicas".

Los participantes relatan un proceso de adaptación, en el cual el compromiso con las actividades y su evolución fueron confirmándolos en sus asociaciones respectivas. De diferentes regiones de Ecuador, los participantes tuvieron la oportunidad de ser propuestos y ellos mismos promoverse para la delegación que participaría en Football for Hope 2014. El proceso, explicado por los propios participantes, y ratificado por los responsables, tuvo tres niveles. La primera etapa, una selección interna de la asociación Fudela, a partir del desempeño y las cualidades particulares de cada participante y sobre todo, el plan social que cada uno debió presentar con el énfasis en la captación de la oportunidad para un impacto y una inserción posterior. La segunda fase fue el punto de vista de un Comité, integrado por Fudela y otros miembros externos vinculados a la Embajada del Reino Unido, el deporte y el ámbito universitario, encargados de calificar a los aspirantes a partir de una serie de reuniones presenciales. Finalmente, el apoyo recibido para cada participante de su plan en redes sociales.

Entre los proyectos concebidos se destacan la organización de encuentros barriales con la metodología del fútbol 3 tiempos, la generación de espacios mixtos y la 


\section{El Fútbol en Tres Tiempos Sociales: Observaciones de Un Festival Asociativo en Caju, Río de}

Janeiro, Brasil 2014

extensión a otras actividades culturales como la danza y la música como se observó en el cuadro I. De acuerdo con la persona responsable de la delegación, la elección de cada participante se dio con base en "su compromiso con los proyectos presentados, con las comunidades donde viven, su motivación, su comportamiento evaluado permanentemente en los procesos formativos y su liderazgo". A este respecto, "sus compañeros de procesos también jugaron un rol importante, pues pedimos que también recomienden a quienes ellos consideraban deberían ir, como Embajadores de FUDELA y del país".

El Participante 6 explicó en su entrevista que los cursos y talleres que recibió en su formación incrementaron su confianza: "Siento que lo pude lograr. Mi meta era viajar al festival pero todo esto es parte de un trabajo de perseverancia". En lo que se refieren a los efectos de su compromiso, la Participante 2 dice: "Soy el orgullo de mi familia, de mi madre y de mis hermanos". Otro de los participantes precisa no tener familia directa, solamente cuenta con su abuela e indica que su organización es su familia. Frente a ellos se siente responsable y deseoso de mostrar progresos.

Ya con un paso avanzado, en tanto asiduos participantes en los proyectos locales, cada delegación sumó dos jóvenes líderes, seleccionados para recibir capacitación y asistir en la mediación de los equipos en el festival. La joven líder de Ecuador relató el hecho que: "Aprendí a recibir indicaciones en los talleres y a moderar mi temperamento. Eso me sirvió para trasladar otra actitud a mi casa, no gritar, aceptar otros puntos de visita y al mismo tiempo dar el mío con serenidad, pero con firmeza". De la misma manera, la extensión de los talleres abarcó tópicos como la formación para micro-emprendimientos. La joven líder explica: "Hace unos meses desarrollé mi propio negocio de estética y peluquería. Todo eso es parte de la capacitación y apoyo que recibí”.

Los testimonios expresados en torno a la construcción de una imagen personal positiva, forman parte de lo que Goffman (1959) denomina la presentación del yo en sociedad, es decir, la proyección que hace el individuo en una situación de comunicación, en la cual aspira a generar una imagen para su interlocutor y para sí mismo. En el caso de los participantes entrevistados es posible percibir el esfuerzo por trasmitir su cambio personal, su convicción y sus deseos de continuar con su formación.

En ese sentido, el festival se presentó como un eslabón entre la construcción de sus proyectos. Es interesante reproducir, sin embargo, alguno de los extractos de la etnografía realizada durante el desarrollo de la cita, para luego, volver a presentar las miradas de los jugadores respecto a sus expectativas.

\section{SEGUNDO TIEMPO: OBSERVACIÓN DE LA EXPERIENCIA DURANTE EL FESTIVAL}

Los jóvenes líderes llegaron unos días antes del festival para recibir talleres sobre liderazgo, animaciones, mediación y presentaciones sobre proyectos en desarrollo entre miembros de la red internacional de asociaciones participantes. En cuanto a los jugadores, también pudieron aclimatarse durante

\footnotetext{
${ }^{4}$ En inglés, el término es self.
}

unos días de actividades recreativas y formativas antes de entrar en el festival. Diferentes salas fuera de los terrenos de juego estaban preparadas para los primeros tiempos y otras para los acuerdos en torno a la asignación de puntos posteriores a los encuentros.

Los primeros momentos, donde prevalecen fotos y cantos animados por los mediadores, retratan encuentros con el resto de las delegaciones. Sin embargo, a medida que se acercan los partidos se pone en práctica la metodología de los 3 Tiempos. Todo cobra una dimensión internacional. El festival constituye en sí una experiencia muy intensa, en la cual se envuelven muchas emociones para los participantes. Lo aprendido durante los meses anteriores y la capacidad para llevarlo adelante se ponen a prueba. Al comenzar los partidos el clima de las delegaciones muestra entusiasmo y participación en la definición de las reglas. Diferentes reglas técnicas, inherentes a la práctica del fútbol, como el saque de bandas con la mano o con el pie, o los tiros de esquina son rápidamente consensuadas y no generan mayores problemas. La observación da cuenta de la incorporación de criterios de género en el sentido que la inclusión de las jugadoras implica que deben participar de las acciones para que un gol sea válido, o que su gol vale doble.

Los partidos son intensos, pero los participantes se esfuerzan por cumplir las reglas. Gestos de cortesía y de celebración a cada gol del equipo contrario caracterizan los momentos observados en Caju 2014. La regulación de los encuentros, sin árbitros en el campo de juego, demuestra la incorporación en los jugadores de la dinámica. Los mediadores intervienen en algunos instantes para sostener interpretaciones ante dudas. Pero son los participantes quienes deben resolver en cuestión de segundos situaciones en las cuales deben interpretar las reglas definidas. En un partido observado, los equipos decidieron establecer el silencio como regla, un marco inhabitual en el mundo del fútbol, es decir, que los jugadores solo podían comunicarse a través de gestos, no podían reclamar ningún tipo de situación y no podían gritar.

Durante el festival, los participantes efectivamente son protagonistas del escenario. Varios de ellos son solicitados para reportajes, incluidos algunos televisivos como dos participantes de la delegación de Ecuador ${ }^{5}$. Durante los primeros días, la emoción de estar viviendo tal acontecimiento no deja descansar a varios de los integrantes. Sin embargo, el ritmo se vuelve demasiado intenso por momentos. Los participantes sienten la necesidad de más horas de descanso, inclusive de dormir un poco más, como confiesan en algunas conversaciones. Esto se incrementa a medida que avanzan los días.

Es frecuente que en estos tipos de festivales, la agenda de los participantes esté sobre-cargada con actividades y horarios, al igual de lo que Magee (2011) ha podido constatar en su seguimiento del mundial Homeless en la ciudad de Graz en el año 2003. Pocas horas de sueño, más la suma de algún resultado no esperado puede generar insatisfacciones (Magee \& Jeanes, 2013). En un contexto de

\footnotetext{
${ }^{5}$ Una cadena de televisión de cable acompañó a varias delegaciones latinoamericanas en su preparación y vivencia del festival. En ese recorrido, escogió dos participantes de Ecuador. Estos reportajes se encuentran disponibles en youtube, con el nombre de El Otro Sueño.
} 
alta intensidad emocional, algunos elementos desestabilizadores se hicieron naturalmente presentes en festival de Caju 2014 para la delegación acompañada.

Algunos participantes sintieron y expresaron en conversaciones posteriores a sus entrevistas que uno de los miembros de la delegación se encontraba desganado(a), sin concentración y la suficiente energía para asumir los partidos.

\section{Partido -Segundo Tiempo: delegación de Francia y una delegación de Brasil}

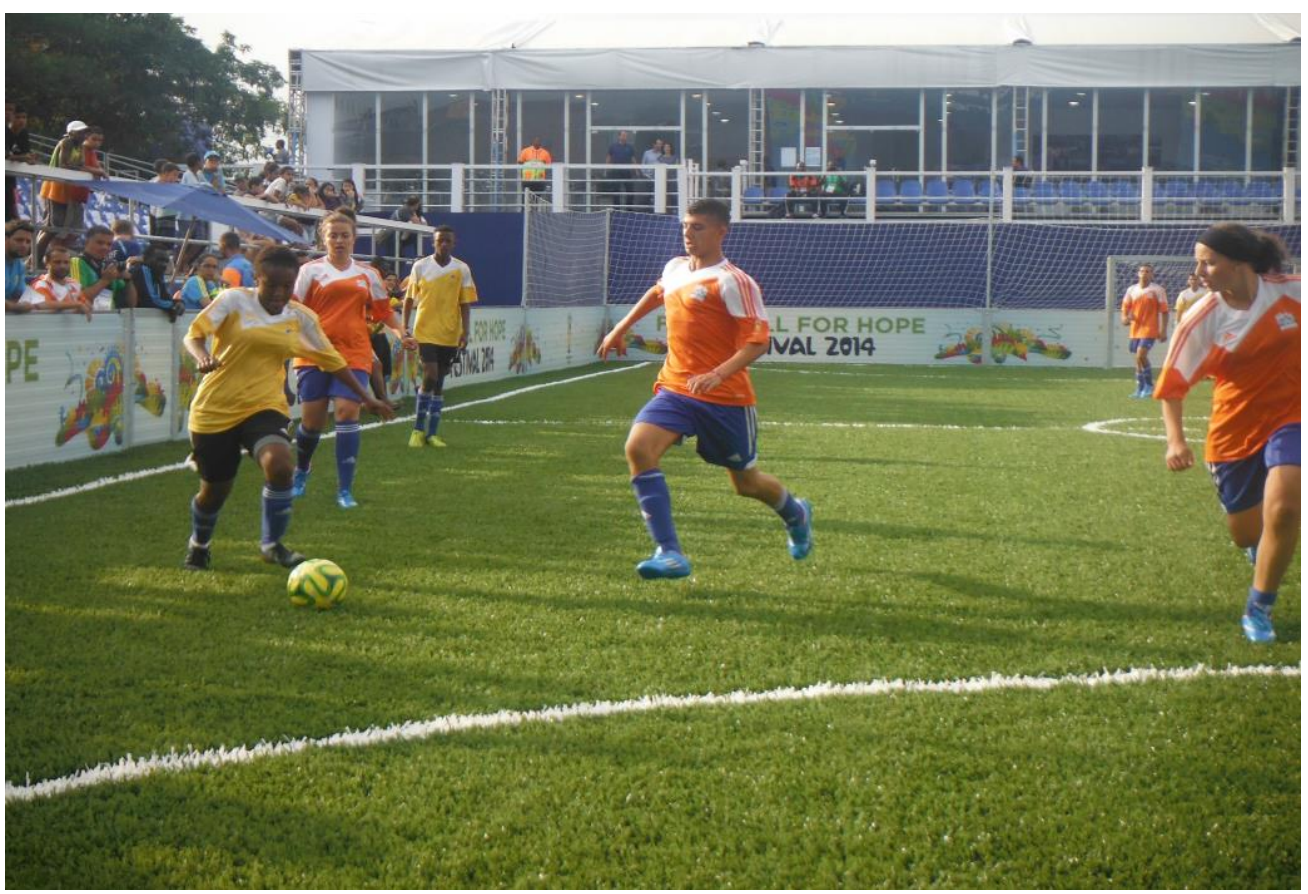

Foto: Autores

Vale notar, que si bien la metodología Fútbol en 3 Tiempos promueve el diálogo, el respeto a las reglas y la concertación, es posible esbozar la hipótesis que si bien los participantes incorporan el espíritu y la reglas, proponerlas, negociarlas, aplicarlas, interpretarlas y discutirlas en la asignación de puntos, varios equipos tienden a construir estrategias variadas para buscar la victoria. Esto significó, en encuentros observados, que los equipos buscan desplegar el mejor fútbol posible, con una alta intensidad de volumen de juego, en un marco de atención a las reglas definidas, pero con una preocupación constante por el marcador. Así, se notan las peticiones de algunas reglas estratégicas en relación con las habilidades o condición física de los equipos, como por ejemplo que los equipos con jugadores de estatura importante proponen doble puntaje por los goles de cabeza. 


\section{Imagen de un Tercer tiempo en el Festival Caju 2014}

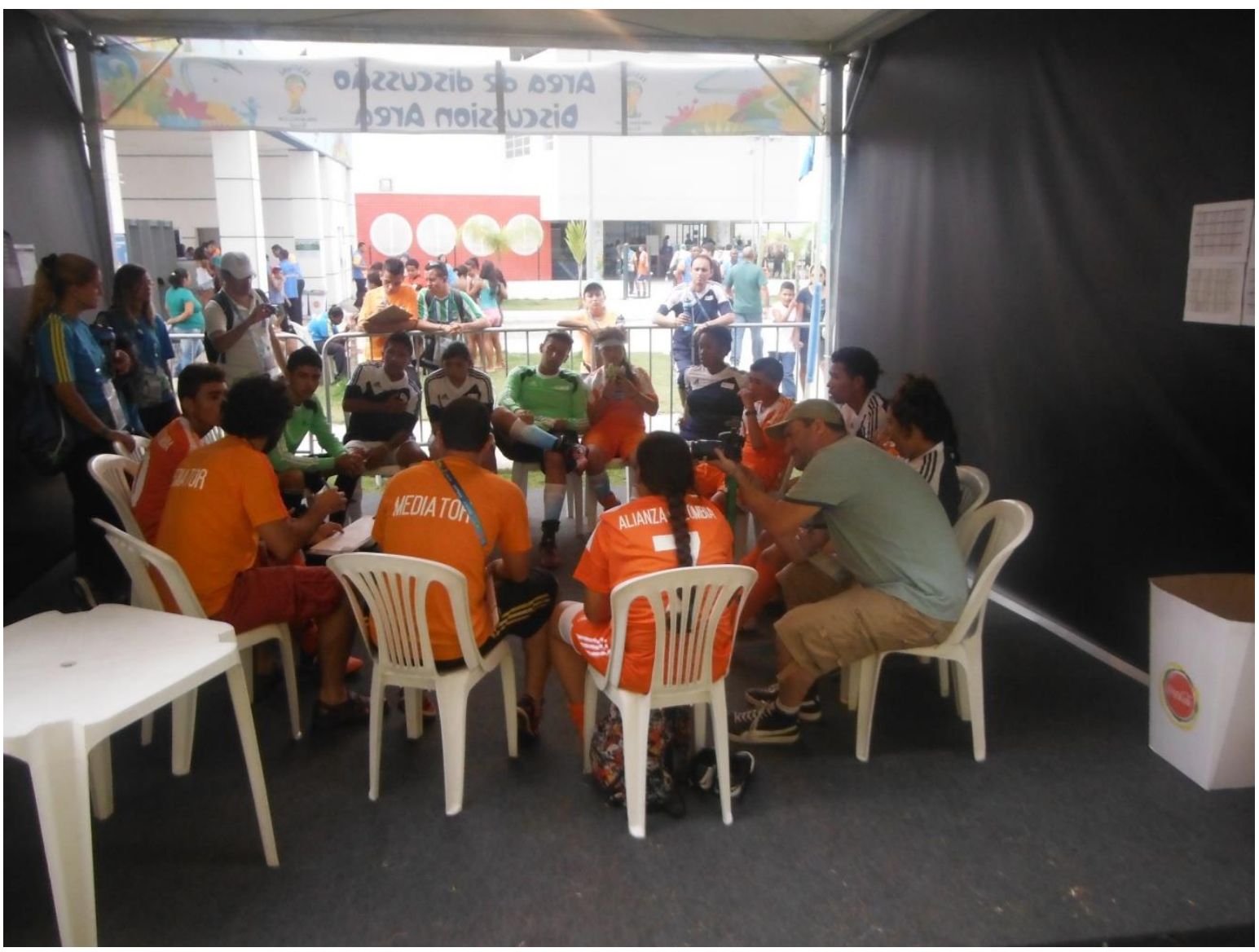

Foto: Autores

El fútbol, en la manera de concebirse, a pesar de ser un fútbol diferente, envuelve una serie de tensiones que Norbert Elias (1992) ha identificado como la (1) polaridad entre dos equipos adversos, (2) la polaridad entre el ataque y la defensa, (3) la polaridad entre la tensión y la cooperación entre los dos equipos rivales y (4) la polaridad entre la competición y la cooperación al interior de cada equipo. De la misma manera que una investigación anterior en el festival Football for Hope 2010 subrayó disparidades de niveles entre algunos equipos (Gannett et al, 2014), malentendidos y abuso de algunas delegaciones para usar las reglas a su favor, un altercado observado en el festival 2014 a partir de la interpretación de una acción, dejó entrever la ansiedad de algunas delegaciones por ganar el encuentro. En un escenario donde ambos equipos precisaban sumar puntos para avanzar a la siguiente etapa y ante un gol del equipo contrario, la objeción del equipo ecuatoriano fue que ninguna jugadora había de la acción. Del otro lado, el argumento era que la jugada había sido iniciada por una participante. Así, el gol fue convalidado en el campo pero no aceptado. El debate se prolongó en el espacio destinado al tercer tiempo y el equipo ecuatoriano, no contento con la situación y la presión ejercida por sus pares para convalidar un gol, no otorgó la cantidad máxima de puntos fair-play a sus oponentes. Estos últimos continuaron pidiendo explicaciones ya que consideraban que merecían la totalidad de puntos.

Este tipo de momentos, donde los participantes se sienten a disgusto con el resultado deportivo y con la interpretación de alguna situación, es decir, del marco referencial (Goffman, 1974), constituye un desafío para los acompañantes de la delegación. Sumada la dosis de serenidad que deben aportar al grupo, ellos forman parte de la coproducción de lo vivido y de la interpretación que se debe dar a determinadas situaciones en aras de aprendizajes más amplios.

Otro momento realmente interesante, e inclusive distinto a todo lo observado, fue la iniciativa entre la delegación de Ecuador y la de Lesoto, Kick4Life, de mezclar los participantes, es decir, que en cada equipo hubiera participantes de ambas delegaciones. La dinámica del encuentro implicó un quiebre con la ansiedad competitiva e introdujo un clima de convivencia y disfrute diferente a otros encuentros. Ante cada acción, la reacción eran los 
aplausos, risas y festejos, ante jugadas llevadas a cabo o incluso aquellas malogradas.

Ahora bien, el desarrollo del festival no puede reducirse a las interacciones acontecidas durante los momentos deportivos, precedidos por los espacios de definición de reglas y extendidos al debate de los terceros tiempos. Los tiempos libres, las visitas turísticas previstas en el programa y los instantes de las comidas abren la oportunidad de dialogar con pares de veinticinco países. El Participante 4 detalla que su particular interés en escuchar y aprender acerca de los proyectos de reciclaje de los que tuvo conocimiento por sus pares de Brasil y de un programa de mejora del medio ambiente en Kenia, en el cual la práctica del fútbol se complementa con acciones de mejora del medio ambiente, recolección de basura y plantación de árboles. Para él: "Este tipo de ideas las puedo proponer en Ecuador para el proyecto que quiero desarrollar y que tuve que presentar para postularme a este festival. Me llevo varias ideas de aquí".

En el mismo sentido de actividades recreativas, varios participantes de diferentes delegaciones expresaban su deseo de acercase a alguna playa de Río de Janeiro, una actividad que por cuestiones de prevención y seguridad no fue prevista por los organizadores. Este deseo fue cumplido, al finalizar el festival, para la delegación de Ecuador.

Con esta descripción del marco del festival, es preciso considerar las proyecciones de los participantes acerca de sus expectativas derivadas, como parte de un tercer momento en sus recorridos.

\section{TERCER TIEMPO: PERSPECTIVAS A FUTURO EN LOS PARTICIPANTES}

Las expectativas derivadas del festival en referencia a los proyectos de los participantes resultan variadas, sin embargo, el común denominador se articula a partir de sus concepciones del fútbol como herramienta social como se identificó en el cuadro I. La Participante 2 manifestó prever desarrollar talleres en su barrio, sobre Deporte y Liderazgo juvenil. El Participante 6 , dice querer incluir en su proyecto de charlas el flagelo de la trata de personas y la explotación juvenil con el esquema de convocatorias asociadas a encuentros recreativos de fútbol.

Consultados al respecto, la organización de campeonatos barriales bajo la metodología de los 3 Tiempos, proyecta el conocimiento de los participantes acerca de las reglas técnicas, pero también la escala de dificultades en su aplicación. El Participante 4 explica: "Existe una modalidad que usamos, pero que no fue aplicada en este festival, que consiste a jugar tomados de la mano, entre chicos y chicas para desplazarnos en los partidos. Es muy complicado pero sirve para la integración de género".

Entre dos de los miembros de la delegación, el proyecto conjunto es el desarrollo de talleres de deporte, baile, a cargo de la participante y buscar asociados para incluir espacios de música. La participante explica la pertinencia de su idea: "Conozco a los chicos de mi barrio, quiero que varios se acerquen al deporte, quiero ser un ejemplo para otros y para otras. Mi experiencia puede servir de ejemplo".

Dos de los participantes, uno de los chicos y una de ellas manifestaron sus deseos de convertirse en futbolistas. En particular, el participante aludía: "Siempre he querido ser futbolista. Me levanto todos los días y salgo a correr, hago 300 abdominales y flexiones. Sólo espero tener la oportunidad de ingresar a un club". Su opinión referida a este programa y a la metodología 3 tiempos considera que: "Me ha ayudado a corregir mi temperamento, a incrementar mi confianza en los otros y a tener paciencia".

$\mathrm{Al}$ no haber sido posible hacer un seguimiento en el tiempo detenido e individual de cada uno de estos participantes consultados en el festival de Саји 2014, los autores realizaron varias consultas con los responsables de la delegación para conocer las situaciones de los jóvenes, a un año del viaje a Río de Janeiro. Algunos participantes avanzaron y mostraron logros considerables mientras que otros y se alejaron de la órbita de la asociación.

Como muestra el cuadro II a continuación, uno de los participantes replanteó sus deseos de estudios, y luego del acompañamiento y las tutorías brindadas, consiguió entrar a la carrera de medicina en la Universidad de San Francisco de Quito, con beca completa por parte de la institución. El mismo sigue vinculado a Fudela, como facilitador de grupos y motivador de jóvenes. Dos de las participantes terminaron la enseñanza secundaria y siguen cerca de la fundación para recibir asesorías sobre la metodología 3Tiempos y su aplicación, llevando adelante lo que expresaron en las entrevistas realizadas en Caju 2014. Una de ellas estudia Gerencia en Liderazgo en la Universidad Salesiana, con beca parcial. De acuerdo con la directora de Fudela, ambas han organizando varias actividades en sus barrios con esta metodología.

Otro de los participantes, ingresó a trabajar en Fudela como colaborador con remuneración donde se desempeña como facilitador de jóvenes, ha realizado un plan social en una escuela, convertido en proyecto institucional de la organización y cuenta con una beca parcial en la Universidad de las Américas en Quito. 


\section{Cuadro II. Situación de los participantes 24 meses después del Festival}

\begin{tabular}{|c|c|c|c|c|c|c|}
\hline & $\begin{array}{c}\text { Participante } \\
1 \\
\end{array}$ & $\begin{array}{c}\text { Participante } \\
2 \\
\end{array}$ & $\begin{array}{c}\text { Participante } \\
3 \\
\end{array}$ & $\begin{array}{c}\text { Participante } \\
4 \\
\end{array}$ & $\begin{array}{c}\text { Participante } \\
5\end{array}$ & $\begin{array}{c}\text { Participante } \\
6 \\
\end{array}$ \\
\hline $\begin{array}{l}\text { En contacto con } \\
\text { la asociación }\end{array}$ & $\begin{array}{c}\text { Con } \\
\text { frecuencia }\end{array}$ & $\begin{array}{c}\text { Con } \\
\text { frecuencia }\end{array}$ & Ocasional & Ocasional & Permanente & $\begin{array}{l}\text { Con } \\
\text { frecuencia }\end{array}$ \\
\hline $\begin{array}{l}\text { Participación en } \\
\text { actividades de } \\
\text { la metodología }\end{array}$ & $\begin{array}{l}\text { Si. Replica la } \\
\text { metodología } \\
\text { en eventos } \\
\text { específicos en } \\
\text { su Provincia. }\end{array}$ & $\begin{array}{l}\text { Si. } \\
\text { Mediadora y } \\
\text { participante } \\
\text { en eventos } \\
\text { específicos }\end{array}$ & $\begin{array}{l}\text { Ocasional en } \\
\text { el año } 2015 .\end{array}$ & $\begin{array}{l}\text { No ha sido } \\
\text { activo en } \\
2015 \text { y } 2016\end{array}$ & $\begin{array}{l}\text { Permanente. Trabaja } \\
\text { en Fudela, en el } \\
\text { ámbito } \\
\text { administrativo- } \\
\text { financiero. Apoya } \\
\text { como Facilitador y } \\
\text { mediador en procesos } \\
\text { y eventos específicos }\end{array}$ & $\begin{array}{l}\text { Si. } \\
\text { Mediador y } \\
\text { participante } \\
\text { en eventos } \\
\text { específicos }\end{array}$ \\
\hline $\begin{array}{c}\text { Estatus de } \\
\text { voluntario, o } \\
\text { miembro de la } \\
\text { fundación }\end{array}$ & $\begin{array}{l}\text { Joven Líder } \\
\text { voluntaria }\end{array}$ & $\begin{array}{l}\text { Joven Líder } \\
\text { referente } \\
\text { Voluntaria }\end{array}$ & $\begin{array}{l}\text { Ocasional en } \\
\text { el año } 2015 . \\
\text { No ha } \\
\text { participado } \\
\text { en } 2016 .\end{array}$ & $\begin{array}{l}\text { Ocasional en } \\
\text { el año } 2015 . \\
\text { No ha } \\
\text { participado } \\
\text { en } 2016 .\end{array}$ & $\begin{array}{l}\text { Parte del equipo de } \\
\text { trabajo de FUDELA. } \\
\text { Posición remunerada. }\end{array}$ & $\begin{array}{l}\text { Joven Líder } \\
\text { Voluntario }\end{array}$ \\
\hline $\begin{array}{c}\text { Avances en sus } \\
\text { estudios }\end{array}$ & $\begin{array}{l}\text { Terminó el } \\
\text { bachillerato } \\
\text { en } 2015 . \text { En } \\
\text { análisis de } \\
\text { opciones de } \\
\text { estudiar para } \\
\text { algún oficio } \\
\text { específico. }\end{array}$ & $\begin{array}{l}\text { Estudiando } \\
\text { en } 2016 \text { en } \\
\text { Universidad } \\
\text { Salesiana - } \\
\text { con beca. } \\
\text { Estudia } \\
\text { Gerencia en } \\
\text { Liderazgo }\end{array}$ & $\begin{array}{l}\text { Concluyendo } \\
\text { en } 2916 \mathrm{su} \\
\text { bachillerato, } \\
\text { en último } \\
\text { año. }\end{array}$ & $\begin{array}{l}\text { Está } \\
\text { concluyendo } \\
\text { el } \\
\text { bachillerato, } \\
\text { en su último } \\
\text { año, } 2016 .\end{array}$ & $\begin{array}{l}\text { Estudiando en } 2016 \\
\text { en Universidad de las } \\
\text { Américas UDLA. } \\
\text { Con beca parcial. } \\
\text { Comunicación }\end{array}$ & $\begin{array}{l}\text { Estudiando } \\
\text { desde } 2015 \text { en } \\
\text { la Universidad } \\
\text { San Francisco } \\
\text { de Quito } \\
\text { USFQ. Con } \\
\text { beca completa } \\
\text { Estudia } \\
\text { Medicina } \\
\end{array}$ \\
\hline $\begin{array}{c}\text { Salto a otro } \\
\text { nivel de fútbol }\end{array}$ & No & No & No & $\begin{array}{l}\text { Se probó en } \\
\text { un equipo } \\
\text { profesional, } \\
\text { no fue } \\
\text { retenido y } \\
\text { luego probó } \\
\text { suerte en } \\
\text { ligas barriales }\end{array}$ & No & No \\
\hline
\end{tabular}

Construcción: Autores con información de Fudela.

Uno de los jóvenes y una de las chicas se han alejado de la asociación pasados los meses como se ilustra en el cuadro II. Uno de ellos ha seguido con su emprendimiento en su plan social en su barrio, pero ahora vinculado a acciones de la iglesia de la que forma parte de acuerdo con las consultas realizadas respecto a su devenir. Al igual que en otros proyectos donde existen momentos intensos en lo que se refiere a viajes y participación en festivales, muchas pueden ser las razones del alejamiento (Segura M. Trejo, 2013a), entre ellas, las expectativas no cumplidas de los participantes durante el viaje, o aquellas no satisfechas para sí mismo o en relación al papel que esperaban que la asociación debía cumplir en sus planes. Otras veces, se trata simplemente una consecuencia de los vaivenes en la vida de los participantes, una oportunidad de mudarse a otra ciudad (Segura et al, 2015), o por el contrario, una crisis personal que envuelve ruptura de lazos. En el caso de la participante, la dirección de Fudela indica que se desconectó del proyecto y no tienen noticias de ella a un año y medio del festival. Las tuvieron durante unos meses mientras la llamaban pero, poco a poco, fue perdiéndose el lazo. La observación etnográfica realizada ubica este dato como poco sorprendente, dado que se percibió en esta participante un grado de desconcentración durante el desarrollo del festival. Inclusive algunos de sus compañeros manifestaron molestias frente a esa impresión. Sobre su futuro, si bien la participante tuvo que presentar un proyecto social, confesó que no sabía "exactamente cómo lo iba a poner en funcionamiento".

Finalmente, la joven líder, iniciadora de su emprendimiento antes del viaje a Brasil en 2014, continúa con su trabajo y participa del plan social que diagramó para su comunidad a partir de las informaciones brindadas por Fudela.

A partir de este estimativo de la temporalidad pos-festival, es necesario insistir en el énfasis de los participantes a medida que el tiempo transcurre, tanto de aquellos que quedaron en contacto, para discernir cuáles son las características que permitieron fortalecer los lazos como aquellos, que por distintitos motivos pueden haberse alejado. En ambos escenarios puede haber razones que indiquen avances, o retrocesos, tanto en una $u$ otra circunstancia. 


\section{El Fútbol en Tres Tiempos Sociales: Observaciones de Un Festival Asociativo en Caju, Río de}

Janeiro, Brasil 2014

Con estos avances, sobre los cuales se seguirá recabando informaciones, hallazgos y reflexiones a partir de la participación en festivales y encuentros in situ, así como su seguimiento, se desprenden a continuación una serie de consideraciones finales para este escrito.

\section{Discusión Post Festival}

El análisis de las percepciones y las observaciones recabadas durante el festival de Caju 2014 Football for Hope indica que la vivencia de los participantes se inscribe en un marco de referencia (Goffman, 1974), en el que, al menos momentáneamente, los participantes proyectan una parte de su identidad, sus aspiraciones y una manera de presentarse en sociedad (Goffman, 1959). El esfuerzo realizado para llegar al festival forma parte de una narrativa de cambios en sus vidas y de una motivación que hasta el instante del festival constituye un objetivo muy importante.

La dinámica in situ, basada en la metodología Fútbol 3 Tiempos, a pesar de ser pensada para superar la idea de un éxito anclado en la victoria o la derrota deportiva, mostró diferentes facetas en la observación etnográfica realizada en el festival. Algunos participantes y varias delegaciones, aun familiares de antemano con la metodología, trasmitían ansiedad para usarla a su favor y ganar los encuentros como un objetivo primordial. Precisamente, la clave del éxito en términos de inclusión en referencia a los programas que usan el deporte para propósitos sociales consiste en la desestructuración de la dimensión competitiva (Stherchele, 2015), es decir, en la introducción de lógicas y dinámicas que integren la recreación y la solidaridad en vez del objetivo deportivo como horizonte en el plano de los participantes.

Sin embargo, es interesante confirmar que el contexto de este fútbol en tres tiempos, ofrece la perspectiva de una dinámica mixta. Esto puede posibilitar múltiples situaciones y oportunidades para trabajar la cuestión de género en varias dimensiones. Resta a explorar, con más discernimiento, cómo funciona, cuáles son las ventajas y cuáles son los límites de este espacio mixto. La indagación de las actividades de esta red internacional de asociaciones, de la cual Fudela forma parte, permitió a los autores enterarse de la realización durante septiembre de 2015 de la siguiente edición del Festival Latinoamericano de Fútbol 3 Tiempos en Santiago de Chile. En él se puso en práctica la dinámica de mesclar equipos de diferentes delegaciones en el campo de juego, una manera de quebrar la ansiedad competitiva. A este encuentro asistió uno de los participantes de Ecuador en Caju 2014.

La participación en este tipo de festivales, como fue el caso de los participantes en Caju 2014 se ubica como un momento cumbre de una etapa de esmero, destaque y motivaciones en sus trayectoria. La vivencia es intensa, por momentos agotadora y deja abierto el interrogante de saber qué sucede con ellos en los meses, inclusive en los años posteriores a esta experiencia y cuál ha sido el impacto de tal experiencia en sus vidas.

La mayoría de las investigaciones realizadas se focalizan en las vivencias de los participantes en los festivales observados (Sherry, 2010; Magee, 2011; Magee \& Jeanes, 2013; Gannett et al, 2014), no obstante, existe un déficit evidente, de investigaciones independientes, sobre el devenir de los jugadores y el impacto, estimado no únicamente en indicadores pensados por los organizadores de los eventos, sino por la estimación y las percepciones de los participantes acerca de la experiencia en retrospectiva (Segura et al, 2015). Los propios autores se encuentran comprometidos con observaciones de campo y son conscientes que estas reflexiones merecen más atención y diferentes enfoques. Además, es importante trascender el intervalo de los festivales e indagar acerca de la continuidad de los proyectos y las vidas de los participantes. ¿Qué pasa con ellos? ¿Cómo puede potencializarse la participación en un determinado festival o foro con un esquema de desarrollo personal pensado a más largo aliento?

Es natural que algunos participantes muestren evoluciones ejemplares, sin embargo, otros se alejan. Es necesario, entonces, recurrir a esfuerzos metodológicos para conocer estos casos y no focalizarse únicamente en las historias de éxito deseadas.

Estos avances de investigación, aquí presentados, dejan en claro que se estamos en presencia de una temática interesante para explorar e interpretar desde las ciencias sociales, en particular desde la sociología. El fútbol, en tanto herramienta social, no puede suplantar déficits en otras áreas sociales, como la educación escolar, posibilidades económicas y/o ausencias familiares. Empero, puede brindar diferentes recursos para el trabajo social y para la construcción personal de los participantes de estos proyectos.

En un contexto más amplio, referido a la crisis mundial que vive el mundo del fútbol y sus instituciones, los escándalos en los cuales varias de sus confederaciones continentales y muchas federaciones nacionales se han visto envueltos, dejan el interrogante de saber si en el futuro se convertirán éstas en instituciones más sensibles para pensar en lógicas diferentes a la hegemonía del negocio. Esto no significa, tampoco, que se vuelquen a hacer "caridad", otorgando subsidios y despreocupándose de la ética y la transparencia. Sin embargo, acercarse un poco más a las metodologías y las propuestas que han surgido desde la esfera de las organizaciones no gubernamentales podría ser materia de apoyo y de reflexión. La investigación en ciencias sociales tendrá también su papel en este horizonte. 
Un último aspecto a ser apunto en la discusión propuesta por este artículo, se refiere al hecho de poder producir, también, investigaciones y publicaciones relevantes en idioma español y en portugués. La mayoría del caudal científico de las investigaciones publicadas en esta área se encuentran en los journals en inglés, una tendencia en la cual estos autores han buscado participar (Segura et al, 2015). No obstante, es importante más allá del campo de conocimiento académico, que los investigadores dejen un legado a las organizaciones con las cuales desarrollan sus observaciones y que pueda ser accesible no sólo a los responsables, sino a los participantes también. En este caso, la investigación puntual fue realizada con una delegación de Ecuador y se optó, por lo tanto, escribir los primeros avances en español como parte de un legado y un posible enriquecimiento de las reflexiones con la organización y su entorno.

\section{REFERENCIAS}

Archetti, E. (2003), Masculinidades: fútbol, tango y polo en la Argentina, Buenos Aires: Antropofagia.

Attali, M. (2004), Le Sport et ses valeurs, Paris: La Dispute.

Becker, H. (1960), "Notes on the concepts of commitment", American Journal of Sociology, 66(1): 32-40.

Becker, H. (1963), Outsiders, Studies of the Sociology of Deviance, New York: The Free Press.

Burnett, C. (2006), "Building social capital through an active community club", International Review for the Sociology of Sport, 41(3): 283-294.

Burnett, C. (2009), "Engaging sport-fordevelopment for social impact in the South African context, Sport in Society: Cultures, Commerce, Media, Politics, 12(9): 1192-1205.

Coalter, F. (2010), "The politics of sport-fordevelopment: Limited focus programmes and broad gauge problems", International Review for the Sociology of Sport, 45(3): 295-314.

Elias, N. (1992), "La dynamique des groupes sportifs et 1'exemple du football", Sport et Civilisation. La violence maîtrisée, Elias, Norbert \& Eric Dunning, Paris: Fayard.

Gannett, K., Kauffman, Z., Clark, M. \& McGarvey, S. (2014), "Football with three "halves": A qualitative exploratory study of the football3 model at the Football for Hope Festival 2010", Journal of Sport for Development, 2(3): 1-12.

Gasparini, W. \& Vieille-Marchiset, G. (2008), Le Sport dans les quartiers: pratiques sociales et politiques publiques, Paris : PUF.

Giulianotti, R. (2005), Sport : a critical sociology, Cambridge : Polity Press.

Giulianotti, R. (2011), "The Sport, Development and Peace Sector: A Model of Four Social Policy Domains", Journal of Social Policy, 40(4): 757-776.

Goffman, E. (1959), The Presentation of Self in Everyday Life, Harmondsworth: Penguin.

Goffman, E. (1974), Frame Analysis: An Essay on the Organization of Social Experience, Londres: Harper \& Row.

Kidd, B. (2008), “A new social movement: Sport for development and peace", Sport in Society: Cultures, Commerce, Media, Politics 11(4): 370-380.

Lofland, J. \& Lofland, L. (1995), Analyzing social settings, a guide to qualitative observation and analysis, Belmonth: Wadsworth.

Magee, J. (2011), "Disengagement, demotivation, vulnerable groups and sporting inclusion: A case study of the Homeless World Cup", Soccer \& Society 12(2): 159-173.

Magee, J. \& Jeanes, R. (2013), "Football's coming home: A critical evaluation of the Homeless World Cup as an intervention to combat social exclusion", International Review for the Sociology of Sport 48(1): 3-19.

Jeanes, R. (2013), "Education through sport? Examining HIV / Aids education and sport-fordevelopment through the perspective of Zambian young people", Sport, Education and Society, 18(3): $388-406$

Rookwood, J. \& Palmer, C. (2011), "Invasion games in war-torn nations: Can football help to build peace?", Soccer \& Society 12(2): 184-200.

Segura M. Trejo, F. (2011), La Homeless World Cup et le Championnat de lutte contre l'exclusion sociale en France. Analyse sociologique de parcours d'exception, Tese para doutorado em sociologia, Paris: Escola de Altos Estudos em Ciências Sociais de Paris.

Segura M. Trejo, F. (2013a), “A Ball can change the world: Percepciones y situaciones en el Mundial de 
los Desamparados, la Homeless World Cup, una revisión crítica desde la vivencia y la mirada de los jugadores", Acta Sociológica, UNAM 60 (1): 103-127.

Segura M. Trejo, F. (2013b), "Football experiences to combat social exclusion and a World Cup: what kind of social capital beyond the tournament?", Documento de trabajo División de Administración Pública, CIDE, 287: 1-42.

Segura M. Trejo, F. (2014a), "O uso do futebol social como ferramenta internacional", Ciência $e$ Cultura, 66(2): 31-34.

Segura M. Trejo, F. (2014b) “Apuntes sobre la utilización del fútbol como recurso social: Hacia una descripción internacional comparativa", Documento de trabajo División de Administración Pública, CIDE, 285:1-31.

Segura M. Trejo, F. (2014c), "Reflexões sobre o uso do futebol social", En Prêmio Petrobras Esporte Educacional, Centro Integrado de Estudos e Programas de Desenvolvimento Sustentável, Río de Janeiro: 2330 .

Segura, F., Attali, M. \& Magee, J. (2015), “The experience of defeat: Applying Goffman to examine a football tournament for socially excluded homeless individuals", International Review for the Sociology of Sport, On Line Fist, $27^{\text {th }}$ October: 1-16.

Sherry, E. (2010), "(Re)engaging marginalized groups through sport: The Homeless World Cup", International Review for the Sociology of Sport 45(1): 59-71.
Sherry, F. \& O’May, F. (2013), “Exploring the impact of sport participation in the Homeless World Cup on individuals with substance abuse or mental health disorders", Journal of Sport for Development 1(2): 1-9.

Spaiij, R. (2009), "Sport as a vehicle for social mobility and regulation of disadvantaged urban youth: lessons from Rotterdam", The International Review for the Sociology of Sport 44: 247-264.

Sterchele, D. (2015), "De-sportizing physical activity: From sport-for-development to play-fordevelopment", European Journal for Sport and Society, 12 (1): 97-120.

Sudgen, J. (2008), “Anyone for football and peace? The challenges of using sport in the service of co-existing in Israel”, Soccer \& Society 9(3): 405-415. 